Christopher Fleischmann*, Irina Leher, David Scherb, Alexander Wolf, Jörg Miehling, Sandro Wartzack, Stefan Sesselmann

\title{
Femoral Shape and Size Variability from segmented CT datasets for patient-specific THA planning
}

\begin{abstract}
Biomechanical functionality of artificial hips strongly correlates with quality of life of patients after total hip arthroplasty. As the numbers of total hip arthroplasty are growing due to increasing life expectancy, biomechanical research is of utmost importance to improve the implants used and the operative procedures applied. Multibody simulation is used to predict forces and moments inside the human body. Generic scaling is usually performed to adapt the human models used in multibody simulation to individual patients. However, since the shape and size of the bones can vary considerably, this type of scaling often is not sufficient. In this work various CT datasets were used to quantify differences of individual femoral shapes, especially with regard to important biomechanical hip parameters, such as the CCD angle or the femoral offset. Our results prove that multibody simulations should be modeled more patientspecific to be able to calculate articular forces and moments more precisely, and thus, to improve surgical planning.
\end{abstract}

Keywords: Total hip arthroplasty, Biomechanics, Segmentation, Multibody simulation, Subject-specific modeling, Surgery planning, Orthopaedics

https://doi.org/10.1515/cdbme-2020-3125

\section{Introduction}

Due to demographic change in sense of a progressively ageing population, cases of osteoarthritis especially of the hip and hence, the numbers of total hip arthroplasty (THA) are

\footnotetext{
${ }^{*}$ Corresponding author: Christopher Fleischmann: Institute for Biomedical Engineering, Ostbayerische Technische Hochschule Amberg-Weiden, Hetzenrichter Weg 15, 92637 Weiden, Germany, e-mail: c.fleischmann@oth-aw.de

Irina Leher, Stefan Sesselmann: Institute for Biomedical Engineering, Ostbayerische Technische Hochschule AmbergWeiden, Hetzenrichter Weg 15, 92637 Weiden, Germany David Scherb, Alexander Wolf, Jörg Miehling, Sandro Wartzack: Engineering Design, Friedrich-Alexander-Universität Erlangen-Nürnberg, Martensstraße 9, 91058 Erlangen, Germany
}

rising [1]. THA ensures the mobility of patients and thus, increases their quality of life [2].

Although THA was designated as one of the most successful operations of the 20th century, with a life rate of up to $95 \%$ after 10 years in patients older than 75 years, an increasing number of revision surgeries is expected in nearer future $[3,4]$.

Revision THA is characterized by the fact that it does not improve pain and function as well as primary THA $[5,6]$.

Hence, meticulous planning of hip surgery parameters, such as the femoral centrum-collum-diaphyseal (CCD) angle or the acetabular anteversion is of utmost importance to ensure functionality in case of revision THA. However, joint forces and moments as well as other important biomechanical factors are not considered in THA planning at all.

Multibody simulation (MBS) is essential to correctly estimate these parameters and hence, to improve surgical planning. MBS allow to simulate forces, moments and muscle activities in order to gain important information about human biomechanics [7, 8].

Models of the human body consisting of rigid bodies (bones), muscles and joints are mostly used for MBS purpose. In order to adapt these models individually to patients, height and weight can be adjusted. These adjustments are usually based on generic scaling.

However, important patient-specific information is usually lost or not simulated correctly. The purpose of this work is to quantify individual differences of important landmarks of the femur bone for biomechanical simulation to demonstrate the importance of an individualized adaption of these landmarks to human models. Due to adjustments of models to individual femur physiology, the muscle attachment points of the MBS-model and in consequence the calculated forces and moments of the hip joint will change [9].

\section{Material and Methods}

For this study, 7 CT-datasets were examined. Therefore, the DICOM-images were imported to Materialise Mimics 
Research (Materialise NV, Leuven, Belgium). First, important geometrical parameters with influence on the biomechanical function such as the CCD angle or the femoral offset were determined in 2D anatomical planes (Fig 1a). Second, the bony structures of the CT data were segmented. Then the femur was separated from the pelvis (Fig 1b) and a $3 \mathrm{D}$ reconstruction of the femur was performed (Fig 1c). Four different landmarks were set to the $3 \mathrm{D}$ femur model on the femoral center of rotation (COR), the proximal posterior greater trochanter point (PPTP), posterior femoral head point (PFHP) and the anterior femoral head point (AFHP). Distances were then measured between the COR and PPTP (d-COR-PPTP) and the PFHP and the AFHP (d-PFHPAFHP).

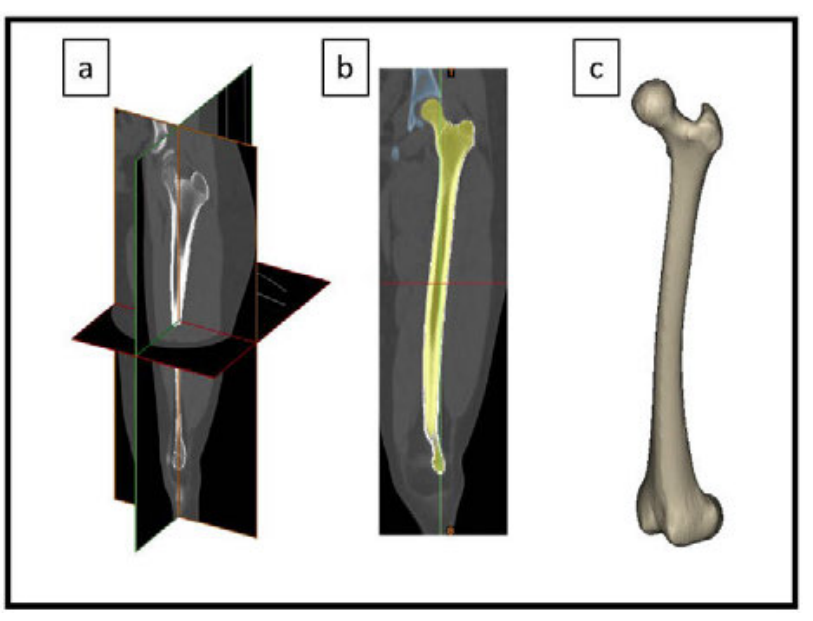

Figure 1: Segmentation workflow: a) Imported DICOM-Files of the CT-Scan b) Marking of bony structures and differntiation of pelvis (blue) and the femur (yellow); c) 3D reconstruction of the femur.

\section{Results}

The results of the measurements of the CCD angle and the femoral offset are shown in Table 1. The minimum measured CCD angle was $118.3^{\circ}$ and the maximum angle was $139.5^{\circ}$. The mean value of the measurements gave a CCD angle of $130.1^{\circ} \pm 7.2$.

The femoral offset also showed significantly different values. The lowest femoral offset was $29.7 \mathrm{~mm}$ and the maximum $46.8 \mathrm{~mm}$. The mean offset was $37.8 \mathrm{~mm} \pm 6.8$ for the seven patients.

The mimimal distance of the d-COR-PPTP was $42.7 \mathrm{~mm}$ and the maximum $56.7 \mathrm{~mm}$. The mean was $51.0 \mathrm{~mm} \pm 4.4$. The distances for d-PFHP-AFHP were $34.0 \mathrm{~mm}$ (minimum), $49.9 \mathrm{~mm}$ (maximum) with a mean value of $43.9 \mathrm{~mm} \pm 5.2$.
Table 1: Minimum, maximum and mean values of the CCD-angle and the femoral-offset for the 7 patients.

\begin{tabular}{llll}
\hline & Min & Max & Mean $( \pm$ SD) \\
\hline CCD-angle $\left[^{\circ}\right]$ & 118.3 & 139.5 & $130.1( \pm 7.2)$ \\
Femoral-offset [mm] & 29.7 & 46.8 & $37.8( \pm 6.8)$ \\
d-COR-PPTP [mm] & 42.7 & 56.7 & $51.0( \pm 4.4)$ \\
d-PFHP-AFHP [mm] & 34.0 & 49.4 & $43.9( \pm 5.2)$ \\
\hline
\end{tabular}

\section{Discussion}

The results show large differences for all measured parameters. If these different shapes and sizes of the femur were considered in a patient-specific way in MBS, muscle and tendon attachment points would change and thus, lead to more precise moments and forces of the hip joint. As a result, more realistic results of MBS could lead to a better understanding of hip biomechanics and thus, help to improve surgical planning of THA.

\section{Conclusion}

The large differences in the shape and size of bones and the need of patient-specific modelling have also been shown in other studies [10]. Differences in size and shape of the femur lead to changes in the tendon and muscle attachment points and hence, to more precise results in MBS.

\section{Author Statement}

Research funding: The author state no funding involved. Conflict of interest: Authors state no conflict of interest.

\section{References}

[1] Pabinger C, Geissler A. Utilization rates of hip arthroplasty in OECD countries. Osteoarthritis Cartilage. 2014 Jun;22(6):734-41.

[2] Mariconda M, Galasso O, Costa GG, Recano P, Cerbasi S. Quality of life and functionality after total hip arthroplasty: a long-term follow-up study. BMC Musculoskelet Disord. 2011;12:222. Published 2011 Oct 6.

[3] Learmonth ID, Young C, Rorabeck C. The operation of the century: total hip replacement. Lancet. 2007 Oct 27;370(9597):1508-19. Review.

[4] Rajaee SS, Campbell JC, Mirocha J, Paiement GD. Increasing Burden of Total Hip Arthroplasty Revisions in Patients Between 45 and 64 Years of Age. J Bone Joint Surg Am. 2018 Mar 21;100(6):449-458. 
[5] Evans JT, Evans JP, Walker RW, Blom AW, Whitehouse MR, Sayers A. How long does a hip replacement last? A systematic review and meta-analysis of case series and national registry reports with more than 15 years of follow-up. Lancet. 2019;393(10172):647-654.

[6] Vanhegan IS, Malik AK, Jayakumar P, UI Islam S, Haddad FS. A financial analysis of revision hip arthroplasty: the economic burden in relation to the national tariff. Bone Joint J. 2012;94:619-623.

[7] Miehling J., Forst R., Wartzack S., Sesselmann S.:

Biomechanischer Simulator zur prä-operativen Abschätzung und Optimierung der post-operativen Muskelfunktion am Beispiel der Revisionsendoprothetik des Hüftgelenks. Deutscher Kongress für Orthopädie und Unfallchirurgie (DKOU2016, Berlin, 25. Oktober 2016 - 28. Oktober 2016)
[8] Sesselmann, S., Miehling, J., Wartzack, S. \& Forst, R. (2016). Enhancement of Surgical Planning through PatientSpecific Biomechanical Modeling and Simulation. - 37th SICOT Orthopaedic World Congress - Rome 2016.

[9] Scherb, D., Wolf, A., Fleischmann, C., Sesselmann, S., Wartzack, S., Miehling, J.: Conceptual Approach to estimate the musculoskeletal follow-ups of endoprosthetic hip replacements. CAMS-Knee OpenSim Workshop - Zürich 2020.

[10] Tkany L., Hofstätter B., Petersik A., Miehling J., Wartzack S., Sesselmann S.: A new design process for anatomically enhanced osteosynthesis plates. Journal of Orthopaedic Research (2019). 